\title{
Entropic Repulsion of the Lattice Free Field, II. The 0-Boundary Case
}

\section{Jean-Dominique Deuschel}

Fachbereich Mathematik, Technische Universität Berlin, D-10623 Berlin, Germany.

E-mail: deuschel@math.tu-berlin.de

Received: 20 November 1995/Accepted: 17 April 1996

\begin{abstract}
This paper is a continuation of [5]. We consider the Euclidean massless free field on a box $V_{N}$ of volume $N^{d}$ with 0 -boundary condition; that is the centered Gaussian field with covariances given by the Green function of the simple random walk on $\mathbb{Z}^{d}, d \geqq 3$, killed as it exits $V_{N}$. We show that the probability, that all the spins are positive in the box $V_{N}$ decays exponentially at a surface rate $N^{d-1}$. This is in contrast with the rate $N^{d-2} \log N$ for the infinite field of [5].
\end{abstract}

\section{Introduction}

The object of this paper is to analyze the asymptotical behavior of a Gibbsian Gaussian field, under the condition that the variables are positive in a large finite box. These asymptotics play an important role in the construction of droplets on a "hard surface", cf. $[1,6,10]$, and in related questions dealing with quasi-locality, cf. [7], and entropic repulsion [7, 11].

More precisely, let $\Lambda=[-1,1]^{d}$ be the unit box in $\mathbb{R}^{d}$ and set $V_{N}=N \Lambda \cap \mathbb{Z}^{d}$. Next consider the Gaussian field $P_{N}^{0}$ on $\Omega_{N}=\mathbb{R}^{V_{N}}$ with density with respect to the Lebesgue measure $\lambda_{N}(d X)=\prod_{i \in V_{N}} d X(i)$ of the form

$$
P_{N}^{0}(d X)=\frac{1}{Z_{N}} \exp \left(-\frac{1}{2} \sum_{\{i, j\} \cap V_{N} \neq \emptyset} Q_{\mathrm{d}}(i, j)(X(i)-X(j))^{2}\right) \lambda_{N}(d X),
$$

where $Z_{N}$ is a normalizing constant, $Q_{\mathrm{d}}(i, j)=\frac{1}{2 d} 1_{|i-j|=1}$ is the transition matrix of the simple random walk on $\mathbb{Z}^{d}$, and we set $X(j)=0$ for $j \notin V_{N}$. Thus the spins are "tied down" at the boundary of $V_{N} . P_{N}^{0}$ can be viewed as the finite Gibbs distribution on $\Omega_{N}$ to the nearest neighbor quadratic interaction

$$
\mathscr{J}=\left\{J_{\{i, j\}}(X)=Q_{\mathrm{d}}(i, j)(X(i)-X(j))^{2},\{i, j\} \subseteq \mathbb{Z}^{d}\right\}
$$

with 0 -boundary conditions on $V_{N}^{\complement}$. We will be working in the transient dimensions $d \geqq 3$; then $P_{N}^{0}$ converges weakly to $P^{0}$, the infinite Gibbs distribution, sometimes called (discrete) Euclidean massless free field. $P^{0}$ is the centered Gaussian field on 
$\Omega=\mathbb{R}^{\mathbb{Z}^{d}}$ with covariance matrix $G$, the Green function of the simple random walk in $\mathbb{Z}^{d}$, cf. [8].

Let

$$
\Omega_{N}^{+}=\left\{X \in \Omega_{N}: X(k) \geqq 0, k \in V_{N}\right\} .
$$

In a previous paper with E. Bolthausen and O. Zeitouni, we have shown,

$$
\lim _{N \rightarrow \infty} \frac{1}{N^{d-2} \log N} \log P^{0}\left(\Omega_{N}^{+}\right)=-2 \mathbf{G} \mathbf{C}^{\prime},
$$

where $\mathbf{G}=\lim _{N \rightarrow \infty} E_{N}^{0}\left[X(0)^{2}\right]=E^{0}\left[X(0)^{2}\right]$ and $\mathbf{C}^{\prime}=\operatorname{cap}_{\mathbb{R}^{d}}(\Lambda)$ is the Newtonian capacity of $\Lambda$ in $\mathbb{R}^{d}$, cf. [5]. The presence of the $\log N$ factor in the exponent, is best explained by the fact that, under the "hard wall" condition $\Omega_{N}^{+}$, the spins are repelled to the height $\sqrt{4 \mathrm{G} \log N}$ as $N \rightarrow \infty$, cf. Prop. 1.3 of [5] or (1.6) below.

In this paper we replace the infinite Gibbs measure $P^{0}$ by the finite Gibbs measure $P_{N}^{0}$ in (1.2). In particular we describe the effect of the 0-boundary condition on the entropic repulsion. We differentiate between two regimes, depending whether one looks

inside the box, i.e. far from the boundary: $\Omega_{\delta N}^{+}$for some $\delta \in(0,1)$,

or

$$
\text { up to the boundary: } \Omega_{N}^{+} \text {. }
$$

In the first regime (1.3), we have a convergence very similar to (1.2):

$$
\lim _{N \rightarrow \infty} \frac{1}{N^{d-2} \log N} \log P_{N}^{0}\left(\Omega_{\delta N}^{+}\right)=-2 \mathbf{G C}(\delta),
$$

where $\mathbf{C}(\delta)=\operatorname{cap}_{\Lambda}(\delta \Lambda)$ is the Newtonian capacity of $\delta \Lambda=[-\delta, \delta]^{d}$ in $\Lambda$, cf. Theorem 2.2 below, and the same entropic repulsion as in [5]:

$$
\begin{aligned}
& \lim _{N \rightarrow \infty} \sup _{k \in V_{\delta N}} P_{N}^{0}\left(X(k) \leqq \sqrt{a \log N} \mid \Omega_{N}^{+}\right) \\
& \quad=\lim _{N \rightarrow \infty} \sup _{k \in V_{\delta N}} P_{N}^{0}\left(X(k) \geqq \sqrt{b \log N} \mid \Omega_{N}^{+}\right)=0,
\end{aligned}
$$

for each $a<4 \mathbf{G}<b$ and $\delta \in(0,1)$.

Note that $\mathbf{C}(\delta)=O\left((1-\delta)^{-1}\right)$ as $\delta \uparrow 1$, so that we expect a faster decay for $\delta=1$. This is due to the 0 -boundary condition, which makes it less likely for the variable to be positive. In fact, in the second regime (1.4), we have a surface order which can be interpreted as a purely boundary effect: let $\partial_{L} V_{N}=V_{N} \backslash V_{N-L}=\{k \in$ $\left.V_{N}: \operatorname{dist}\left(k, V_{N}^{\complement}\right)<L\right\}$, and set

$$
\partial_{L} \Omega_{N}^{+} \equiv\left\{X \in \Omega_{N}: X(i) \geqq 0, i \in \partial_{L} V_{N}\right\},
$$

then we show in our main result, Theorem 4.1,

$$
\lim _{N \rightarrow \infty} \frac{1}{N^{d-1}} \log P_{N}^{0}\left(\Omega_{N}^{+}\right)=\lim _{L \rightarrow \infty} \lim _{N \rightarrow \infty} \frac{1}{N^{d-1}} \log P_{N}^{0}\left(\partial_{L} \Omega_{N}^{+}\right)=-\sum_{i=-d}^{d} \kappa^{0}\left(e_{i}\right),
$$

where $\kappa^{0}\left(e_{i}\right)$ is a certain "surface tension" in the direction of the $i^{\text {th }}$ unit vector $e_{i}$ in $\mathbb{R}^{d}$. 
The major tool in the derivation of (1.7) is the following interpolation in the "intermediate regime": let $\left\{L_{N}, N \in \mathbb{N}\right\}$ be a monotone increasing sequence with $2 \leqq L_{N}$ and $\lim _{N \rightarrow \infty} \frac{L_{N}}{N}=0$, then

$$
\begin{aligned}
-\infty & <\liminf _{N \rightarrow \infty} \frac{L_{N}}{N^{d-1} \log L_{N}} \log P_{N}^{0}\left(\Omega_{\left(N-L_{N}\right)}^{+}\right) \\
& \leqq \limsup _{N \rightarrow \infty} \frac{L_{N}}{N^{d-1} \log L_{N}} \log P_{N}^{0}\left(\Omega_{\left(N-L_{N}\right)}^{+}\right)<0,
\end{aligned}
$$

cf. Prop. 2.5 and 2.9. In fact, we can show that, under the condition $\Omega_{N}^{+}$, we have at distance $L_{N}$ from the boundary of $V_{N}$ an entropic repulsion of the order $O\left(\sqrt{\log L_{N}}\right)$.

The rest of the paper is divided into 4 sections. Section 2 gives a proof of (1.5) and (1.8). Our main tool is the random walk representation of the covariance of $P_{N}^{0}$ and a conditioning argument. In Sect. 3 we prove the entropic repulsion (1.6), here the argument is based on the FKG property of the conditional field $P_{N}^{0}\left(\cdot \mid \Omega_{N}^{+}\right)$. Section 4 deals with the convergence (1.7) in the boundary regime. Finally, the Appendix contains some useful estimates for the random walk.

Before concluding, let us state two important remarks. First it should be noted that the above results can be easily generalized to arbitrary finite range interactions $Q$ and fixed boundary conditions $a \in \Omega$, cf. $[5,1]$. That is, in the definition (1.1) of $P_{N}^{0}$, we can replace $Q_{\mathrm{d}}$ by the positive finite range matrix $Q$ of an irreducible symmetric random walk on $Z^{d}$ and set $X(k)=a(k), k \notin V_{N}$. In particular, using monotonicity one can show that, for any log-tempered $a \in \Omega,(1.5)$ and (1.6) hold with the same constants ${ }^{1} \mathbf{C}(\delta)$ and $\mathbf{G}$, cf. Remark 2.4. Also (1.7) is true for any constant boundary condition $a(k) \equiv a \in \mathbb{R}, k \in \mathbb{Z}^{d}$, with $\kappa^{0}\left(e_{i}\right)$ replaced by the corresponding $\kappa^{a}\left(e_{i}\right)$.

Second, much of what we have discussed above holds with some modifications for the recurrent dimension $d=2$. The main difference here is the logarithmic divergence of the variance $G_{N}(0,0)=O(\log N)$ as $N \rightarrow \infty$. This of course implies that the infinite measure $P^{0}$ does not exist. We will treat this case in a separate paper with E. Bolthausen. In particular, we show that the boundary behavior (1.7) is the same as for $d \geqq 3$, however, in the interior of the box, we have a $(\log N)^{2}$-decay. More precisely, we show in [4], for each $\delta \in(0,1)$,

$$
\begin{aligned}
-2 \mathbf{G C}(\delta) & \leqq \liminf _{N \rightarrow \infty} \frac{1}{(\log N)^{2}} \log P_{N}^{0}\left(\Omega_{\delta N}^{+}\right) \\
& \leqq \limsup _{N \rightarrow \infty} \frac{1}{(\log N)^{2}} \log P_{N}^{0}\left(\Omega_{\delta N}^{+}\right) \leqq-\frac{1}{2} \mathbf{G C}(\delta),
\end{aligned}
$$

where $\mathbf{C}(\delta)=\operatorname{cap}_{\Lambda}(\delta \Lambda)$ as above, and $\mathbf{G}=\lim _{N \rightarrow \infty} \frac{G_{N}(0,0)}{\log N}$.

\section{The Behavior Inside the Box}

In this section we give a proof of (1.5) and (1.8). Our main tool is the random walk representation of the covariance matrix of $P_{N}^{0}$ : Let $\left\{\xi_{n}: n \in \mathbb{N}_{0}=\mathbb{N} \cup\{0\}\right\}$ be the simple random walk in $\mathbb{Z}^{d}$ generated by $Q_{\mathrm{d}}$. We denote by $\mathbb{P}_{i}$ and $\mathbb{E}_{i}$ the probability

${ }^{1}$ Of course, in case $Q \neq Q_{\mathrm{d}}$, the constant $\mathbf{C}(\delta)$ has to be adapted to the corresponding capacity, cf. [3] 
and expectation for the walk with start at $i \in \mathbb{Z}^{d}$. Let $\tau_{N}=\inf \left\{n \in \mathbb{N}_{0}: \xi_{n} \notin V_{N}\right\}$ be the first exit time of $V_{N}$, then the covariance of $P_{N}^{0}$ is given by

$$
\operatorname{cov}_{P_{N}^{0}}(X(i), X(j))=G_{N}(i, j)=\mathbb{E}_{i}\left[\sum_{n=0}^{\tau_{N}} 1_{j}\left(\xi_{n}\right)\right], \quad i, j \in V_{N},
$$

cf. Appendix of [3]. Let $G(i, j)=\mathbb{E}_{i}\left[\sum_{n=0}^{\infty} 1_{j}\left(\xi_{n}\right)\right], i, j \in \mathbb{Z}^{d}$, be the Green function of the discrete Laplacian, then, for each $\delta \in(0,1)$,

$$
\lim _{N \rightarrow \infty} G_{N}(i, j)=G(i, j) \text { uniformly on } V_{\delta N} \text {. }
$$

This implies the weak convergence $\lim _{N \rightarrow \infty} P_{N}^{0}=P^{0}$, where $P^{0}$, the infinite Gibbs state, is the centered Gaussian field with covariance $G$.

Let us fix some notation: $c_{1}, c_{2}, c_{3}, \ldots \in \mathbb{R}^{+}$are generic constants which do not depend on $N$ or $L_{N}$, but are not necessarily the same at different occurrences. Also for $\Lambda \subset \mathbb{Z}^{d}$ we write

$$
\Omega^{+}(\Lambda)=\{X \in \Omega: X(k) \geqq 0, k \in \Lambda\} .
$$

Our first result is the proof of

Theorem 2.2. Let $\delta \in(0,1)$, then

$$
\lim _{N \rightarrow \infty} \frac{1}{N^{d-2} \log N} \log P_{N}^{0}\left(\Omega_{\delta N}^{+}\right)=-2 \mathbf{G C}(\delta),
$$

where

$$
\mathbf{C}(\delta)=\operatorname{cap}_{\Lambda}(\delta \Lambda)=\inf \left\{\frac{1}{2 d}\|\nabla h\|_{L^{2}(\Lambda)}^{2}: h \in H_{0}^{1}(\Lambda), h \geqq 1_{\delta \Lambda}\right\}
$$

is the Newtonian capacity ${ }^{2}$ of $\delta \Lambda$ in $\Lambda$.

Proof. The proof follows exactly the argument of [5], so that we don't go into details and rather concentrate on the identification of the new constant $\mathbf{C}(\delta)=$ $\operatorname{cap}_{\Lambda}(\delta \Lambda)$, which is alternatively given by

$$
\mathbf{C}(\delta)=\sup \left\{2\left\langle\phi, 1_{\delta \Lambda}\right\rangle_{\Lambda}-\left\langle\phi 1_{\delta \Lambda},\left(\mathfrak{F}_{\Lambda}\left(1_{\delta \Lambda} \phi\right)\right\rangle_{\Lambda}: \phi \in C(\Lambda)\right\},\right.
$$

where $\langle\cdot, \cdot\rangle_{\Lambda}$ is the scalar product in $L^{2}(\Lambda)$ and $\mathfrak{G}_{\Lambda} \phi(x)=\int_{\Lambda} \mathfrak{g}_{\Lambda}(x, y) \phi(y) d y$, is the Green operator associated with the brownian motion, killed as it exits $\Lambda$, cf. [1].

We start with the lower bound

$$
\liminf _{N \rightarrow \infty} \frac{1}{N^{d-2} \log N} \log P_{N}^{0}\left(\Omega_{\delta N}^{+}\right) \geqq-2 \mathbf{G C}(\delta) .
$$

A glance at the proof of [5], shows that the only two new ingredients are, the uniform convergence (2.1), and the convergence of the relative entropy:

$$
\lim _{N \rightarrow \infty} \frac{1}{N^{d-2} \log N} \mathbf{H}_{V_{\delta N}}\left(P_{N}^{a(N)} \mid P_{N}^{0}\right)=\frac{a^{2} \mathbf{C}(\delta)}{2},
$$

\footnotetext{
${ }^{2} H_{0}^{1}(\Lambda)$ is the usual Sobolev space
} 
here $\mathbf{H}_{V_{\delta N}}\left(P_{N}^{a(N)} \mid P_{N}^{0}\right)$ is the relative entropy of $P_{N}^{a(N)}$ with respect to $P_{N}^{0}$ restricted to the box $V_{\delta N}$ and $P_{N}^{a(N)}$ is the Gaussian field on $\Omega_{N}$ with covariance $G_{N}$ and constant mean $E_{N}^{a(N)}[X(k)]=a(N)=\sqrt{a \log N}, k \in V_{N}$, for some $a \in \mathbb{R}^{+}$. In order to prove (2.3), first note the identity

$$
\mathbf{H}_{V_{\delta N}}\left(P_{N}^{a(N)} \mid P_{N}^{0}\right)=\frac{a(N)^{2}}{2}\left\langle 1_{V_{\delta N}}, G_{N, \delta}^{-1} 1_{V_{\delta N}}\right\rangle_{V_{N}}
$$

where $G_{N, \delta}$ is the covariance matrix $G_{N}$ restricted to $V_{\delta N}, G_{N, \delta}^{-1}$ the inverse of $G_{N, \delta}$, and $\langle\cdot, \cdot\rangle_{V_{N}}$ is the scalar product in $\ell^{2}\left(V_{N}\right)$, cf. [3]. Next, $\left\langle 1_{V_{\delta N}}, G_{N, \delta}^{-1} 1_{V_{\delta N}}\right\rangle_{V_{N}}=$ $\operatorname{cap}_{V_{N}}\left(V_{\delta N}\right)$ is the capacity of $V_{\delta N}$ in $V_{N}$ with respect to the simple random walk, and using the same argument as in the proof of Lemma 2.2 of [3], one shows the convergence

$$
\lim _{N \rightarrow \infty} \frac{1}{N^{d-2}} \operatorname{cap}_{V_{N}}\left(V_{\delta N}\right)=\operatorname{cap}_{\Lambda}(\delta \Lambda)
$$

which yields (2.3). As far as the upper bound is concerned:

$$
\limsup _{N \rightarrow \infty} \frac{1}{N^{d-2} \log N} \log P_{N}^{0}\left(\Omega_{\delta N}^{+}\right) \leqq-2 \mathbf{G C}(\delta),
$$

one again uses the convergence (2.1) and the fact that, for each $f \in C_{b}(\Lambda)$, with $f_{N}(k)=f(k / N), k \in V_{N}$,

$$
\lim _{N \rightarrow \infty} N^{-d-2}\left\langle f_{N}, G_{N} f_{N}\right\rangle_{V_{N}}=\left\langle f, \mathfrak{G}_{\Lambda} f\right\rangle_{\Lambda}
$$

cf. [1]. Now the result follows from the equality

$$
\mathbf{C}(\delta)=\sup \left\{\frac{\left\langle 1_{\delta \Lambda}, h\right\rangle_{\Lambda}^{2}}{\left\langle h 1_{\delta \Lambda},\left(\mathfrak{b}_{\Lambda}\left(h 1_{\delta \Lambda}\right)\right\rangle_{\Lambda}\right.}: h \text { piecewise constant on a uniform grid }\right\} \text {. }
$$

Remark 2.4. Note that we do not use explicitly the geometry of $V_{N}$ or $V_{\delta N}$ in the above argument. Also, using monotonicity, we could consider any log-tempered boundary condition $a \in \Omega_{\log }=\left\{a \in \Omega: \lim _{|k| \rightarrow \infty} \frac{|a(k)|^{2}}{\log |k|}=0\right\}$. Thus, let $\Gamma, \Lambda$ be two bounded open domains of $\mathbb{R}^{d}$ with piecewise smooth boundaries. Set

$$
\Gamma_{N}=N \Gamma \cap \mathbb{Z}^{d}, \quad \Lambda_{N}=N \Lambda \cap \mathbb{Z}^{d} \quad \text { and } \quad P_{\Lambda_{N}}^{a}=P^{0}\left(\cdot \mid X(k)=a(k), k \notin \Lambda_{N}\right) .
$$

Then if $\Gamma \subset \Lambda$ with $\operatorname{dist}\left(\Gamma, \Lambda^{\complement}\right)>0$,

$$
\lim _{N \rightarrow \infty} \frac{1}{N^{d-2} \log N} \log P_{\Lambda_{N}}^{a}\left(\Omega^{+}\left(\Gamma_{N}\right)\right)=-2 \operatorname{Gcap}_{\Lambda}(\Gamma),
$$

where $\operatorname{cap}_{\Lambda}(\Gamma)=\inf \left\{\frac{1}{2 d}\|\nabla h\|_{L^{2}(\Lambda)}^{2}: h \in H_{0}^{1}(\Lambda), h \geqq 1_{\Gamma}\right\}$ is the capacity of $\Gamma$ in $\Lambda$.

Our next step is the upper bound in the intermediate regime.

Proposition 2.5. Let $\frac{L_{N}}{N} \searrow 0$ with $L_{N} \geqq 2$, then

$$
\limsup _{N \rightarrow \infty} \frac{L_{N}}{N^{d-1} \log L_{N}} \log P_{N}^{0}\left(\Omega_{\left(N-L_{N}\right)}^{+}\right)<0 .
$$


Proof. Let $W_{N}=W_{N}\left(L_{N}\right)=\left\{k \in V_{N}: L_{N} \leqq \operatorname{dist}\left(k, V_{N}^{\complement}\right) \leqq 2 L_{N}\right\}$, denote by $W_{N}^{\text {o }}$ the odd points of $W_{N}$ and by $W_{N}^{\mathrm{e}}$ the even points in the interior of $W_{N}$. Let $\mathscr{F}_{N}^{\mathrm{o}}=$ $\sigma\left\{X(k): k \in W_{N}^{\text {o }}\right\}$ be the sigma algebra generated by the odd points. By the Markov property of $P_{N}^{0}$, conditioned upon $\mathscr{F}_{N}^{\mathrm{o}}$, the $\left\{X(k), k \in W_{N}^{\mathrm{e}}\right\}$ are independent Gaussian with variance 1 and mean $\bar{X}(k)=\sum_{j} Q_{\mathrm{d}}(k, j) X(j)$, cf. [3]. Thus

$$
\begin{aligned}
P_{N}^{0}\left(\Omega_{\left(N-L_{N}\right)}^{+}\right) & \leqq P_{N}^{0}\left(\Omega^{+}\left(W_{N}\right)\right) \leqq P_{N}^{0}\left(\Omega^{+}\left(W_{N}^{\mathrm{e}}\right) \cap \Omega^{+}\left(W_{N}^{\mathrm{o}}\right)\right) \\
& \leqq E_{N}^{0}\left[P_{N}^{0}\left(\Omega^{+}\left(W_{N}^{\mathrm{e}}\right) \mid \mathscr{F}_{N}^{\mathrm{o}}\right) ; \Omega^{+}\left(W_{N}^{\mathrm{o}}\right)\right] \\
& =E_{N}^{0}\left[\prod_{k \in W_{N}^{\mathrm{e}}}\{1-\phi(\bar{X}(k))\} ; \Omega^{+}\left(W_{N}^{\mathrm{o}}\right)\right]
\end{aligned}
$$

where

$$
\frac{1}{2} e^{-x^{2} / 2} \geqq \phi(x) \equiv(2 \pi)^{-1 / 2} \int_{x}^{\infty} e^{-t^{2} / 2} d t \geqq \frac{1}{2 x} e^{-x^{2} / 2}, \quad x \geqq 2 .
$$

For given $m=m\left(L_{N}\right) \geqq 2$, let $I_{N} \equiv\{k \in: \bar{X}(k) \leqq m\}$ and set $A_{N}=\left\{X:\left|I_{N}\right| \geqq\right.$ $\left.\frac{1}{2}\left|W_{N}^{\mathrm{e}}\right|\right\}$. Then

$$
\begin{aligned}
E_{N}^{0}\left[\prod_{k \in W_{N}^{\mathrm{e}}}\{1-\phi(\bar{X}(k))\} ; \Omega^{+}\left(W_{N}^{\mathrm{o}}\right)\right]= & E_{N}^{0}\left[\prod_{k \in W_{N}^{\mathrm{e}}}\{1-\phi(\bar{X}(k))\} ; \Omega^{+}\left(W_{N}^{\mathrm{o}}\right) \cap A_{N}\right] \\
& +E_{N}^{0}\left[\prod_{k \in W_{N}^{\mathrm{e}}}\{1-\phi(\bar{X}(k))\} ; \Omega^{+}\left(W_{N}^{\mathrm{o}}\right) \cap A_{N}^{\complement}\right] \\
\leqq & (1-\phi(m))^{\frac{1}{2}\left|W_{N}^{\mathrm{e}}\right|}+P_{N}^{0}\left(\Omega^{+}\left(W_{N}^{\mathrm{o}}\right) \cap A_{N}^{\complement}\right) .
\end{aligned}
$$

For the first term, we have, in view of (2.6), the a priori estimate

$$
(1-\phi(m))^{\frac{1}{2}\left|W_{N}^{\mathrm{e}}\right|} \leqq \exp \left(-c_{1} N^{d-1} L_{N} \frac{e^{-m^{2} / 2}}{m}\right) .
$$

On $\Omega^{+}\left(W_{N}^{\mathrm{o}}\right) \cap A_{N}^{\complement}$, we have

$$
\bar{S}_{N}^{\mathrm{e}} \equiv \frac{1}{\left|W_{N}^{\mathrm{e}}\right|} \sum_{k \in W_{N}^{\mathrm{e}}} \bar{X}(k) \geqq \frac{m}{2}, \quad \text { with } P_{N}^{0}\left(\bar{S}_{N}^{\mathrm{e}} \geqq \frac{m}{2}\right) \leqq \exp \left(-\frac{m^{2}}{8 \operatorname{var}\left(\bar{S}_{N}^{\mathrm{e}}\right)}\right),
$$

since $\bar{S}_{N}^{\mathrm{e}}$ is centered Gaussian. Set $S_{N}^{\mathrm{e}}=\frac{1}{\left|W_{N}^{\mathrm{e}}\right|} \sum_{k \in W_{N}^{\mathrm{e}}} X(k)$, then, since $\bar{X}(k)$ is the conditional expectation of $X(k), \operatorname{var}\left(\bar{S}_{N}^{\mathrm{e}}\right) \leqq \operatorname{var}\left(S_{N}^{\mathrm{e}}\right)$ with

$$
\operatorname{var}\left(S_{N}^{\mathrm{e}}\right)=\frac{1}{\left|W_{N}^{\mathrm{e}}\right|^{2}} \sum_{i, j \in W_{N}^{\mathrm{e}}} G_{N}(i, j) \leqq c_{2} \frac{L_{N}}{N^{d-1}}
$$

cf. (A.3) below. This yields

$$
P_{N}^{0}\left(\Omega^{+}\left(W_{N}^{\mathrm{o}}\right) \cap A_{N}^{\mathrm{c}}\right) \leqq \exp \left(-\frac{c_{3} N^{d-1} m^{2}}{L_{N}}\right) .
$$


In view of (2.7) and (2.8) we may choose $m\left(L_{N}\right)=\sqrt{a \log \left(L_{N}\right)}$ for some $0<a<2$ and conclude the proof.

We now turn to the proof of the lower bound in the intermediate regime:

Proposition 2.9. Let $\frac{L_{N}}{N} \searrow 0$ with $L_{N} \geqq 2$, then there exists a constant $K<\infty$ such that

$$
\liminf _{N \rightarrow \infty} \frac{L_{N}}{N^{d-1} \log L_{N}} \log P_{N}^{0}\left(\Omega_{\left(N-L_{N}\right)}^{+}\right) \geqq-K .
$$

Proof. It will be enough to prove the existence of $K^{\prime}<\infty$ such that

$$
\liminf _{N \rightarrow \infty} \frac{L_{N}}{N^{d-1} \log L_{N}} \log P_{N}^{0}\left(\Omega^{+}\left(W_{N}\left(L_{N}\right)\right)\right) \geqq-K^{\prime} .
$$

Namely, once (2.10) is proved, we can cover $V_{\left(N-L_{N}\right)}$ with $\left\{W_{N}\left(2^{\ell} L_{N}\right), \ell=\right.$ $\left.0, \ldots, \ell_{\max }\right\}, \ell_{\max } \leqq-\log \left(L_{N} / N\right) / \log 2$. Then by FKG property, for large $N$,

$$
\begin{aligned}
P_{N}^{0}\left(X(k) \geqq 0, k \in V_{\left(N-L_{N}\right)}\right) & \geqq \prod_{\ell=0}^{\ell_{\max }} P_{N}^{0}\left(X(k) \geqq 0, k \in W_{N}\left(2^{\ell} \delta_{N}\right)\right) \\
& \geqq \exp \left(-2 K^{\prime} \sum_{\ell=0}^{\ell_{\max }} N^{d-1} \frac{\log \left(2^{\ell} L_{N}\right)}{2^{\ell} L_{N}}\right) \\
& \geqq \exp \left(-K N^{d-1} \frac{\log L_{N}}{L_{N}}\right),
\end{aligned}
$$

for some $K<\infty$. In order to prove (2.10), we use a conditioning argument, which is quite different from the proof of the lower bound of [5]: For given $L_{N}$ and $\varepsilon>0$ let

$$
\Lambda_{N}(\varepsilon) \equiv\left\{k: \frac{L_{N}}{2} \leqq \operatorname{dist}\left(k, V_{N}^{\complement}\right) \leqq \frac{5 L_{N}}{2}\right\} \cap\left[\varepsilon L_{N}^{2 / d}\right] \mathbb{Z}^{d},
$$

$\bar{W}_{N}=W_{N}\left(L_{N}\right) \backslash \Lambda_{N}(\varepsilon)$, and set

$$
q_{k}^{N}(j)=\mathbb{P}_{k}\left(\xi_{\tau_{\varepsilon}}=j ; \tau_{\varepsilon}<\tau_{N}\right), \quad j \in \Lambda_{N}(\varepsilon), k \in \bar{W}_{N},
$$

where $\tau_{\varepsilon}=\inf \left\{n \in \mathbb{N}_{0}: \xi_{n} \in \Lambda_{N}(\varepsilon)\right\}$. Note that

$$
\left|\Lambda_{N}(\varepsilon)\right| \leqq \frac{c_{1} N^{d-1}}{\varepsilon^{d} L_{N}}
$$

In the Appendix (Lemma A.9), we show that one can choose $\varepsilon>0$ independently of $N$, such that

$$
\inf _{k \in \bar{W}_{N}} \mathbb{P}_{k}\left(\tau_{\varepsilon}<\tau_{N}\right)=\inf _{k \in \bar{W}_{N}} \sum_{j \in \Lambda_{N}(\varepsilon)} q_{k}^{N}(j) \geqq \frac{1}{2} .
$$

Let $\mathscr{F}_{\Lambda_{N}(\varepsilon)}=\sigma\left\{X(j), j \in \Lambda_{N}(\varepsilon)\right\}$. Then, conditioned upon $\mathscr{F}_{\Lambda_{N}(\varepsilon)},\left\{X(k), k \in \bar{W}_{N}\right\}$ is a Gaussian field with positive covariance and conditional mean

$$
\bar{X}(k)=E_{N}^{0}\left[X(k) \mid \mathscr{F}_{\Lambda_{N}(\varepsilon)}\right]=\sum_{j \in \Lambda_{N}(\varepsilon)} q_{k}^{N}(j) X(j), \quad k \in \bar{W}_{N} .
$$


Thus for given $m=m\left(L_{N}\right) \geqq 2$,

$$
\begin{aligned}
P^{0}\left(\Omega^{+}\left(W_{N}\right)\right) & =E_{N}^{0}\left[P_{N}^{0}\left(\Omega^{+}\left(W_{N}\right) \mid \mathscr{F}_{\Lambda_{N}(\varepsilon)}\right)\right] \\
& \geqq E_{N}^{0}\left[P\left(\Omega^{+}\left(\bar{W}_{N}\right) \mid \mathscr{F}_{\Lambda_{N}(\varepsilon)}\right) ; X(j) \geqq m, j \in \Lambda_{N}(\varepsilon)\right] .
\end{aligned}
$$

In view of (2.11) and (2.12), we have on $\left\{X(j) \geqq m, j \in \Lambda_{N}(\varepsilon)\right\}$, by the FKG property and the fact that $\operatorname{var}\left(X(k) \mid \mathscr{F}_{\Lambda_{N}(\varepsilon)}\right) \leqq G_{N}(k, k) \leqq \mathbf{G}, k \in \bar{W}_{N}$,

$$
\begin{aligned}
P\left(\Omega^{+}\left(\bar{W}_{N}\right) \mid \mathscr{F}_{\Lambda_{N}(\varepsilon)}\right) & \geqq \prod_{k \in \bar{W}_{N}}(1-\phi(\bar{X}(k) / \sqrt{\mathbf{G}})) \\
& \geqq\left(1-\exp \left(-m^{2} / 8 \mathbf{G}\right)\right)^{\left|\bar{W}_{N}\right|} \geqq \exp \left(-c_{2} N^{d-1} L_{N} e^{-m^{2} / 8 \mathbf{G}}\right) .
\end{aligned}
$$

Also, again by FKG, $(2.11)$ and $G_{N}(j, j) \geqq 1, j \in \Lambda_{N}(\varepsilon)$,

$$
P_{N}^{0}\left(X(j) \geqq m, j \in \Lambda_{N}(\varepsilon)\right) \geqq \prod_{j \in \Lambda_{N}(\varepsilon)} P_{N}^{0}(X(j) \geqq m) \geqq \exp \left(-\frac{c_{3} N^{d-1} m^{2}}{\varepsilon^{d} L_{N}}\right) .
$$

Thus

$$
P_{N}^{0}\left(\Omega^{+}\left(W_{N}\right)\right) \geqq \exp \left(-c_{2} N^{d-1} L_{N} e^{-m^{2} / 8 \mathbf{G}}\right) \exp \left(-\frac{c_{3} N^{d-1} m^{2}}{\varepsilon^{d} L_{N}}\right),
$$

and choosing $m\left(L_{N}\right)=\sqrt{b \log L_{N}}$ for some $b>16 \mathbf{G}$ yields (2.10).

Remark 2.13. For $m>0$ and $a \in \Omega$, consider the measure $P_{N}^{a,(m)}$ on $\Omega_{N}$ given by

$$
P_{N}^{a,(m)}(d X)=\frac{1}{Z_{N}^{a,(m)}} \exp \left(-\frac{m}{2} \sum_{k \in V_{N}} X(k)^{2}\right) P_{N}^{a}(d X),
$$

where $Z_{N}^{a,(m)}$ is a normalizing constant. Then, for each tempered

$$
a \in \Omega^{\prime}=\left\{X \in \Omega: \lim _{|k| \rightarrow \infty}|k|^{-\varepsilon}|a(k)|<\infty, \text { for some } \varepsilon>0\right\},
$$

$P_{N}^{a,(m)}$ converges weakly as $N \rightarrow \infty$ to the centered Gaussian measure $P^{(m)}$ with covariance $G^{(m)}=(1+m)^{-1} \sum_{n=0}^{\infty}(1+m)^{-n} Q_{\mathrm{d}}^{n}$, called Euclidean free field with positive mass $m$. In this case the fixed boundary condition plays no role, in particular, one shows that, for each $\delta \in(0,1)$,

$$
\lim _{N \rightarrow \infty} \frac{1}{N^{d}} \log P_{N}^{a,(m)}\left(\Omega_{\delta N}^{+}\right)=\lim _{N \rightarrow \infty} \frac{1}{N^{d}} \log P^{(m)}\left(\Omega_{\delta N}^{+}\right)=-\delta^{d} K(m)
$$

for some $K(m)>0$, cf. Sect. 3 of [5].

\section{The Entropic Repulsion}

The aim of this section is to prove the entropic repulsion (1.6). The crucial step in the proof will be the following FKG property of $P_{N}^{0}\left(\cdot \mid \Omega_{N}^{+}\right)$:

Lemma 3.1. Let $\emptyset \neq V \subset W \subset V_{N}$, then, for all $k \in V$ and $a>0$,

$$
P_{N}^{0}\left(X(k) \geqq a \mid \Omega^{+}(V)\right) \leqq P_{N}^{0}\left(X(k) \geqq a \mid \Omega^{+}(W)\right) .
$$


Moreover

$$
P_{V}^{0}\left(X(k) \geqq a \mid \Omega^{+}(V)\right) \leqq P_{W}^{0}\left(X(k) \geqq a \mid \Omega^{+}(\bar{V})\right),
$$

where $\bar{V}=\left\{k \in \mathbb{Z}^{d}: \operatorname{dist}(k, V) \leqq 1\right\}$ and $P_{W}^{0}=P_{N}^{0}\left(\cdot \mid X(k)=0, k \in V_{N} \backslash W\right)$.

Proof. Note that $\Omega^{+}(W)=\Omega^{+}(V) \cap \Omega^{+}(W \backslash V)$. Thus, since (3.2) is equivalent with

$$
\begin{aligned}
& P_{N}^{0}\left(\{X(k) \geqq a\} \cap \Omega^{+}(W \backslash V) \mid \Omega^{+}(V)\right) \\
& \quad \geqq P_{N}^{0}\left(X(k) \geqq a \mid \Omega^{+}(V)\right) P_{N}^{0}\left(\Omega^{+}(W \backslash V) \mid \Omega^{+}(V)\right),
\end{aligned}
$$

it suffices to show that $P_{N}^{0}\left(\cdot \mid \Omega^{+}(V)\right)$ is positively correlated. We use a simple approximation argument: for $\beta>0$, define

$$
P_{V, N}^{0, \beta}(d X)=\frac{\exp \left(-\beta \sum_{k \in V}|X(k) \wedge 0|^{2}\right)}{Z_{N}(\beta)} P_{N}^{0}(d X),
$$

where $Z_{N}(\beta)$ is a normalizing constant. Then for each $\beta>0$, by Theorem 1.3 of [9], cf. also Sect. 10.6 of this paper, we know that $P_{V, N}^{0, \beta}$ is positively correlated. Moreover, with respect to the weak convergence, we have

$$
\lim _{\beta \rightarrow \infty} P_{V, N}^{0, \beta}=P_{N}^{0}\left(\cdot \mid \Omega^{+}(V)\right) .
$$

This implies (3.2). As for (3.3), let $\partial \bar{V}=\bar{V} \backslash V$ and note that, by continuity and the Markov property,

$$
\begin{aligned}
P_{V}^{0}\left(X(k) \geqq a \mid \Omega^{+}(V)\right) & =P_{W}^{0}\left(X(k) \geqq a \mid \Omega^{+}(\bar{V}), X(j) \leqq 0, j \in \partial \bar{V}\right) \\
& =\lim _{\varepsilon \searrow 0} P_{W}^{0}\left(X(k) \geqq a \mid \Omega^{+}(\bar{V}), X(j) \leqq \varepsilon, j \in \partial \bar{V}\right) .
\end{aligned}
$$

Thus in order to prove (3.3), it suffices to show, for all $\varepsilon>0$,

$$
P_{W}^{0}\left(X(k) \geqq a \mid \Omega^{+}(\bar{V}), X(j) \leqq \varepsilon, j \in \partial \bar{V}\right) \leqq P_{W}^{0}\left(X(k) \geqq a \mid \Omega^{+}(\bar{V})\right) .
$$

This is equivalent with

$$
\begin{aligned}
P_{W}^{0}(X(k) & \left.\geqq a, X(j) \geqq \varepsilon, j \in \partial \bar{V} \mid \Omega^{+}(\bar{V})\right) \\
& \geqq P_{W}^{0}\left(X(k) \geqq a \mid \Omega^{+}(\bar{V})\right) P_{W}^{0}\left(X(j) \geqq \varepsilon, j \in \partial \bar{V} \mid \Omega^{+}(\bar{V})\right),
\end{aligned}
$$

and follows from the positive correlations of the measure $P_{W}^{0}\left(\cdot \mid \Omega^{+}(\bar{V})\right)$.

Remark 3.4. Note that the FKG property of the conditional field $P_{N}^{0}\left(\cdot \mid \Omega_{N}^{+}\right)$, which was implicitly used in Sect. 4 of [5], does not follow immediately from the positive correlations of the original field $P_{N}^{0}$, since $\Omega^{+}(V)$ is not a cylinder set.

We now turn to the entropic repulsion, first inside the box:

Proposition 3.5. Let $\delta \in(0,1)$ and $a<4 \mathbf{G}<b$, then

$$
\begin{aligned}
& \lim _{N \rightarrow \infty} \sup _{k \in V_{\delta N}} P_{N}^{0}\left(X(k) \leqq \sqrt{a \log N} \mid \Omega_{N}^{+}\right) \\
& \quad=\lim _{N \rightarrow \infty} \sup _{k \in V_{\delta N}} P_{N}^{0}\left(X(k) \geqq \sqrt{b \log N} \mid \Omega_{N}^{+}\right)=0 .
\end{aligned}
$$


Proof. Let $b>4 \mathbf{G}$, then, in view of (3.3),

$$
\sup _{k \in V_{\delta N}} P_{N}^{0}\left(X(k) \geqq \sqrt{b \log N} \mid \Omega_{N}^{+}\right) \leqq \sup _{k \in V_{\delta N}} P^{0}\left(X(k) \geqq \sqrt{b \log N} \mid \Omega_{N+1}^{+}\right),
$$

where the RHS converges to 0 as $N \rightarrow \infty$ by Prop. 1.3 of [5]. Next, let $a<4 \mathbf{G}$ and $k \in V_{\delta N}$, then, by (3.2), for each $\delta<\delta^{\prime}<1$,

$$
P_{N}^{0}\left(X(k) \leqq \sqrt{a \log N} \mid \Omega_{N}^{+}\right) \leqq P_{N}^{0}\left(X(k) \leqq \sqrt{a \log N} \mid \Omega_{\delta^{\prime} N}^{+}\right) .
$$

Now using Theorem 2.2 and precisely the same argument as in Sect. 4 of [5] (noticing in particular, that the height of the entropic repulsion in [5] depends on $\mathbf{G}$ only and not on the capacity $\mathbf{C}^{\prime}$ ), one shows that

$$
\lim _{N \rightarrow \infty} \sup _{k \in V_{\delta N}} P_{N}^{0}\left(X(k) \leqq \sqrt{a \log N} \mid \Omega_{\delta^{\prime} N}^{+}\right)=0
$$
where

Next for $\delta \in(0,1)$, let $W_{N, \delta}\left(L_{N}\right)=\bigcup_{i=1}^{d} W_{N, \delta}^{i}\left(L_{N}\right)$ be the "interior" of $W_{N}\left(L_{N}\right)$,

$$
W_{N, \delta}^{i}\left(L_{N}\right)=\left\{k \in W_{N}\left(L_{N}\right):\left|k_{j}\right| \leqq \delta N, j \neq i\right\} .
$$

Proposition 3.7. Let $\frac{L_{N}}{N} \searrow 0$ and $\lim _{N \rightarrow \infty} L_{N}=\infty$, then there exist two constants $0<b \leqq B<\infty$ such that, for all $\delta \in(0,1)$,

$$
\lim _{N \rightarrow \infty} \sup _{k \in W_{N, \delta}\left(L_{N}\right)} P_{N}^{0}\left(X(k) \leqq \sqrt{b \log L_{N}} \mid \Omega_{N}^{+}\right)=0
$$

and

$$
\limsup _{N \rightarrow \infty} \sup _{k \in W_{N}\left(L_{N}\right)} \frac{E_{N}^{0}\left[X(k) \mid \Omega_{N}^{+}\right]}{\sqrt{B \log L_{N}}} \leqq 1 .
$$

Our first step in the proof of (3.8) is the following

Lemma 3.10. Let $\mathbf{L}_{W_{N}} \equiv \frac{1}{\left|W_{N}\right|} \sum_{k \in W_{N}} \delta_{X(k)}$ be the empirical measure of $W_{N}=$ $W_{N}\left(L_{N}\right)$, then there exist $b^{\prime}>0$, such that, for all $\varepsilon \in(0,1)$,

$$
\lim _{N \rightarrow \infty} P_{N}^{0}\left(\mathbf{L}_{W_{N}}\left[0, \sqrt{b^{\prime} \log L_{N}}\right] \geqq \varepsilon \mid \Omega_{N}^{+}\right)=0 .
$$

Proof. First note that by (3.2),

$$
P_{N}^{0}\left(\mathbf{L}_{W_{N}}\left[0, \sqrt{b^{\prime} \log L_{N}}\right] \geqq \varepsilon \mid \Omega_{N}^{+}\right) \leqq P_{N}^{0}\left(\mathbf{L}_{W_{N}}\left[0, \sqrt{b^{\prime} \log L_{N}}\right] \geqq \varepsilon \mid \Omega^{+}\left(W_{N}\right)\right) \text {. }
$$

We follow the argument of the proof of Prop. 4.1 of [5]: let $W_{N}^{\mathrm{e}}$ be the even elements of $W_{N}, \bar{X}(k)=\sum_{j} Q_{\mathrm{d}}(k, j) X(j)$ and

$$
\mathbf{L}_{W_{N}^{\mathrm{e}}}=\frac{1}{\left|W_{N}^{\mathrm{e}}\right|} \sum_{k \in W_{N}^{\mathrm{e}}} \delta_{X(k)}, \quad \overline{\mathbf{L}}_{W_{N}^{\mathrm{e}}}=\frac{1}{\left|W_{N}^{\mathrm{e}}\right|} \sum_{k \in W_{N}^{\mathrm{e}}} \delta_{\bar{X}(k)}
$$


be the corresponding empirical measures. Then, in view of the proof of Theorem 2.2 above, we can choose $b^{\prime}>0$ such that

$$
P_{N}^{0}\left(\overline{\mathbf{L}}_{W_{N}^{\mathrm{e}}}\left[0, \sqrt{b^{\prime} \log L_{N}}\right] \geqq \varepsilon ; \Omega^{+}\left(W_{N}^{\mathrm{o}}\right)\right) \leqq \exp \left(-c_{1} \varepsilon N^{d-1} L_{N}^{c_{2}}\right),
$$

for some $c_{2}>0$. Thus, by Prop. 2.9,

$$
\lim _{N \rightarrow \infty} P_{N}^{0}\left(\overline{\mathbf{L}}_{W_{N}^{\mathrm{e}}}\left[0, \sqrt{b^{\prime} \log L_{N}}\right] \geqq \varepsilon \mid \Omega^{+}\left(W_{N}\right)\right)=0 .
$$

Next, we use the fact that, for each $\varepsilon^{\prime}>0$,

$$
\limsup _{N \rightarrow \infty} \frac{1}{\left|W_{N}^{\mathrm{e}}\right|} \log P_{N}^{0}\left(\frac{1}{\left|W_{N}^{\mathrm{e}}\right|} \sum_{k \in W_{N}^{\mathrm{e}}}|X(k)-\bar{X}(k)| \geqq \varepsilon^{\prime} \sqrt{\log L_{N}}\right)<0,
$$

in order to conclude

$$
\lim _{N \rightarrow \infty} P_{N}^{0}\left(\mathbf{L}_{W_{N}^{\mathrm{e}}}\left[0, \sqrt{b^{\prime} \log L_{N}}\right] \geqq \varepsilon \mid \Omega^{+}\left(W_{N}\right)\right)=0,
$$

cf. Proof of (4.2) in [5]. Now the result follows from (3.11), (3.12) and

$$
\begin{aligned}
\left\{\mathbf{L}_{W_{N}}\left[0, \sqrt{b^{\prime} \log L_{N}}\right] \geqq \varepsilon\right\} & \subseteq\left\{\mathbf{L}_{W_{N}^{\mathrm{e}}}\left[0, \sqrt{b^{\prime} \log L_{N}}\right] \geqq \varepsilon / 2\right\} \\
& \cup\left\{\mathbf{L}_{W_{N}^{\mathrm{o}}}\left[0, \sqrt{b^{\prime} \log L_{N}}\right] \geqq \varepsilon / 2\right\} .
\end{aligned}
$$

Proof of 3.8. It is enough to show, for each $i=1, \ldots, d$ and $\delta \in(0,1)$,

$$
\lim _{N \rightarrow \infty} \sup _{k \in W_{N, \delta}^{i}} P_{N}^{0}\left(X(k) \geqq \sqrt{b \log L_{N}} \mid \Omega_{N}^{+}\right)=0 .
$$

Define $\Lambda_{N}^{i}=\left\{\ell \in \mathbb{Z}^{d}:\left|\ell_{j}\right|<\delta N / 4, j \neq i,\left|\ell_{i}\right|<L_{N} / 4\right\}, \tilde{V}_{N}=\bigcap_{\ell \in \Lambda_{N}^{i}}\left(V_{N}+\ell\right)$. Then, for each $k \in W_{N, \delta}^{i}\left(L_{N}\right), \Lambda_{N}^{i}(k)=\Lambda_{N}^{i}+k \subset \tilde{V}_{N}$ with $\operatorname{dist}\left(\Lambda_{N}^{i}(k), \tilde{V}_{N}^{\complement}\right) \geqq L_{N} / 4$ for large $N$. A simple modification of the above lemma shows that there is $b>0$, such that for each $\varepsilon>0$,

$$
\lim _{N \rightarrow \infty} \tilde{P}_{N}^{0}\left(\mathbf{L}_{\Lambda_{N}^{i}(k)}\left[0, \sqrt{b \log L_{N}}\right] \geqq \varepsilon \mid \tilde{\Omega}_{N}^{+}\right)=0,
$$

where $\tilde{P}_{N}^{0}=P_{\tilde{V}_{N}}^{0}$ and $\tilde{\Omega}_{N}^{+}=\Omega^{+}\left(\tilde{V}_{N}\right)$. On the other hand, by (3.3) for each $\ell \in \Lambda_{N}^{i}$,

$$
\begin{aligned}
P_{N}^{0}\left(X(k) \leqq \sqrt{b \log L_{N}} \mid \Omega_{N}^{+}\right) & =P_{\left(V_{N}+\ell\right)}^{0}\left(X(k+\ell) \leqq \sqrt{b \log L_{N}} \mid \Omega^{+}\left(V_{N}+\ell\right)\right) \\
& \leqq \tilde{P}_{N}^{0}\left(X(k+\ell) \leqq \sqrt{b \log L_{N}} \mid \tilde{\Omega}_{N}^{+}\right) \\
& =\tilde{E}_{N}^{0}\left[1_{X(k+\ell) \leqq \sqrt{b \log L_{N}}} \mid \tilde{\Omega}_{N}^{+}\right] .
\end{aligned}
$$

Thus, taking the average over $\Lambda_{N}^{i}$ yields

$$
\begin{aligned}
P_{N}^{0}\left(X(k) \leqq \sqrt{b \log L_{N}} \mid \Omega_{N}^{+}\right) \leqq & \tilde{E}_{N}^{0}\left[\mathbf{L}_{\Lambda_{N}^{i}(k)}\left[0, \sqrt{b \log L_{N}}\right] \mid \tilde{\Omega}_{N}^{+}\right] \\
\leqq & \varepsilon \tilde{P}_{N}^{0}\left(\mathbf{L}_{\Lambda_{N}^{i}(k)}\left[0, \sqrt{b \log L_{N}}\right] \leqq \varepsilon \mid \tilde{\Omega}_{N}^{+}\right) \\
& +\tilde{P}_{N}^{0}\left(\mathbf{L}_{\Lambda_{N}^{l}(k)}\left[0, \sqrt{b \log L_{N}}\right]>\varepsilon \mid \tilde{\Omega}_{N}^{+}\right)
\end{aligned}
$$

for all $\varepsilon \in(0,1)$, and (3.8) follows from (3.13). 
Proof of 3.9. For $k \in W_{N}\left(L_{N}\right)$, let $i \in\{1, \ldots, d\}$ be such that $N-2 L_{N} \leqq\left|k_{i}\right| \leqq$ $N-L_{N}$. Set $\bar{\Lambda}_{N}^{i}=\left\{\ell \in \mathbb{Z}^{d}: \ell_{i}=0,\left|\ell_{j}\right| \leqq N / 4, j \neq i\right\}, U_{N}=\bigcup_{\ell \in \Lambda_{N}}\left(V_{N}+\ell\right)$ and $\tilde{U}_{N}=\bigcup_{j:|j| \leqq L_{N}}\left(U_{N}+j\right)$. By (3.3) we have, for each $\ell \in \bar{\Lambda}_{N}^{i}$,

$$
\begin{aligned}
E_{N}^{0}\left[X(k) \mid \Omega_{N}^{+}\right] & =E_{\left(V_{N}+\ell\right)}^{0}\left[X(k+\ell) \mid \Omega^{+}\left(V_{N}+\ell\right)\right] \leqq E_{U_{N}}^{0}\left[X(k+\ell) \mid \Omega^{+}\left(U_{N}\right)\right] \\
& \leqq E_{\tilde{U}_{N}}^{0}\left[X(k+\ell) \mid \Omega^{+}\left(\bar{U}_{N}\right)\right]=\tilde{E}_{N}^{0}\left[X(k+\ell) \mid \tilde{\Omega}_{\left(N-L_{N}\right)}^{+}\right]
\end{aligned}
$$

where $\tilde{P}_{N}^{0} \equiv P_{\tilde{U}_{N}}^{0}$ and $\tilde{\Omega}_{\left(N-L_{N}\right)}^{+}=\Omega^{+}\left(\bar{U}_{N}\right)$. Thus

$$
E_{N}^{0}\left[X(k) \mid \Omega_{N}^{+}\right] \leqq \tilde{E}_{N}^{0}\left[S_{\bar{\Lambda}_{N}^{i}(k)} \mid \tilde{\Omega}_{\left(N-L_{N}\right)}^{+}\right]
$$

with $S_{{\overline{\Lambda_{N}}}^{i}(k)}=\frac{1}{\left|\bar{\Lambda}_{N}^{i}\right|} \sum_{\ell \in \bar{\Lambda}_{N}^{\imath}} X(k+\ell)$. For each $\alpha>0$, we have the relative entropy bound

$$
\alpha \tilde{E}_{N}^{0}\left[S_{\bar{\Lambda}_{N}^{i}(k)} \mid \tilde{\Omega}_{\left(N-L_{N}\right)}^{+}\right] \leqq-\log \tilde{P}_{N}^{0}\left(\tilde{\Omega}_{\left(N-L_{N}\right)}^{+}\right)+\frac{\alpha^{2}}{2} \tilde{E}_{N}^{0}\left[S_{\bar{\Lambda}_{N}^{i}(k)}^{2}\right],
$$

cf. Lemma 4.7 of [5]. That is, taking the optimal $\alpha>0$,

$$
\left(\tilde{E}_{N}^{0}\left[S_{\bar{\Lambda}_{N}^{i}(k)} \mid \tilde{\Omega}_{N}^{+}\right]\right)^{2} \leqq-2 \log \tilde{P}_{N}^{0}\left(\tilde{\Omega}_{\left(N-L_{N}\right)}^{+}\right) \tilde{E}_{N}^{0}\left[S_{\bar{\Lambda}_{N}^{i}(k)}^{2}\right] .
$$

In the Appendix, we show that

$$
\tilde{E}_{N}^{0}\left[S_{\bar{\Lambda}_{N}^{i}(k)}^{2}\right]=\frac{1}{\left|\bar{\Lambda}_{N}^{i}\right|^{2}} \sum_{\ell, j \in \bar{\Lambda}_{N}^{\imath}} \tilde{G}_{N}(\ell+k, j+k) \leqq c_{1} \frac{L_{N}}{N^{d-1}},
$$

where $\tilde{G}_{N}$ is the covariance associated with $\tilde{P}_{N}^{0}$, cf. (A.3). Also, in view of Prop. 2.9 above, we can find $\tilde{K}<\infty$, such that

$$
\liminf _{N \rightarrow \infty} \frac{L_{N}}{N^{d-1} \log L_{N}} \log \tilde{P}_{N}^{0}\left(\tilde{\Omega}_{\left(N-L_{N}\right)}^{+}\right) \geqq-\tilde{K} .
$$

Now, (3.9) follows from (3.14), (3.15) and (3.16).

\section{Behavior at the Boundary}

In this section we study the behavior of the conditional field $P_{N}^{0}\left(\cdot \mid \Omega_{N}^{+}\right)$close to the boundary of $V_{N}$. In order to formulate the main result, it will be useful to move the boundary of $V_{N}$ to the origin. Thus, let $e_{i}$ denote the $i^{\text {th }}$ unit vector in $\mathbb{R}^{d}$ and write $e_{-i}=-e_{i}, i=1, \ldots, d$. Next, let $\mathbb{Z}_{i}^{d}=\left\{k \in \mathbb{Z}^{d}: k \cdot e_{i}<0\right\}, P^{i, 0}=P^{0}(\cdot \mid X(k)=$ $\left.0, k \notin \mathbb{Z}_{i}^{d}\right)$ and

$$
\partial_{L}^{i} V_{N}=\left\{k \in \mathbb{Z}^{d}:-L-1 \leqq k \cdot e_{i}<0,\left|k_{j}\right| \leqq N, j \neq i\right\} .
$$

Theorem 4.1. For each $i=-d, \ldots, d$ and $L \in \mathbb{N}$ the following limits ${ }^{3}$ $\lim _{N \rightarrow \infty} \frac{1}{N^{d-1}} \log P^{i, 0}\left(\Omega^{+}\left(\partial_{L}^{i} V_{N}\right)\right)=-\kappa_{L}^{0}\left(e_{i}\right)$ with $0<\kappa^{0}\left(e_{i}\right) \equiv \lim _{L \rightarrow \infty} \kappa_{L}^{0}\left(e_{i}\right)<\infty$

\footnotetext{
${ }^{3}$ Actually, $\kappa^{o}\left(e_{l}\right)$ does not depend on $i$, since $Q_{\mathrm{d}}$ is isotropic
} 
exist. Moreover

$$
\lim _{N \rightarrow \infty} \frac{1}{N^{d-1}} \log P_{N}^{0}\left(\Omega_{N}^{+}\right)=\lim _{L \rightarrow \infty} \lim _{N \rightarrow \infty} \frac{1}{N^{d-1}} \log P_{N}^{0}\left(\partial_{L} \Omega_{N}^{+}\right)=-\sum_{i=-d}^{d} \kappa^{0}\left(e_{i}\right) .
$$

The proof of (4.2) requires some additional notation. Let $V_{N}^{i}=\left\{k \in \mathbb{Z}^{d}:-2 N-\right.$ $\left.1 \leqq k \cdot e_{i}<0,\left|k_{j}\right| \leqq N, i \neq j\right\}$. Next, consider the centered Gaussian field $P_{N}^{i, 0}=$ $P^{0}\left(\cdot \mid X(k)=0, k \neq V_{N}^{i}\right)$ with covariances $G_{N}^{i}(k, j)=\mathbb{E}_{k}\left[\sum_{n=0}^{\tau_{N}^{i}} 1_{j}\left(\xi_{n}\right)\right]$, where $\tau_{N}^{i}=$ $\inf \left\{n \geqq 0: \xi_{n} \notin V_{N}^{i}\right\}$. Then $P_{N}^{i, 0}$ converges weakly to $P^{i, 0}$, the centered Gaussian field with covariance

$$
G^{i}(k, j)=\mathbb{E}_{k}\left[\sum_{n=0}^{\tau^{i}} 1_{j}\left(\xi_{n}\right)\right], \quad \text { where } \tau^{i}=\inf \left\{n \geqq 0: \xi_{n} \notin \mathbb{Z}_{i}^{d}\right\} .
$$

The main step in the proof of (4.2), is to show that we can replace $P_{N}^{0}$ by $P^{i, 0}$ :

Lemma 4.3. For each $L \in \mathbb{N}$, we have

$$
\liminf _{N \rightarrow \infty} \frac{1}{N^{d-1}} \log P_{N}^{0}\left(\partial_{L} \Omega_{N}^{+}\right) \geqq \sum_{i=-d}^{d} \liminf _{N \rightarrow \infty} \frac{1}{N^{d-1}} \log P^{0, i}\left(\Omega^{+}\left(\partial_{L}^{i} V_{N}\right)\right),
$$

and

$$
\limsup _{N \rightarrow \infty} \frac{1}{N^{d-1}} \log P_{N}^{0}\left(\partial_{L} \Omega_{N}^{+}\right) \leqq \sum_{i=-d}^{d} \limsup _{N \rightarrow \infty} \frac{1}{N^{d-1}} \log P^{0, i}\left(\Omega^{+}\left(\partial_{L}^{i} V_{N}\right)\right) .
$$

Proof. Set $\hat{\partial}_{L}^{i} V_{N}=\left\{k \in \partial_{L} V_{N}: N-L \leqq e_{i} \cdot k \leqq N\right\}$, then $\Omega^{+}\left(\partial_{L} V_{N}\right)=\bigcap_{i=-d}^{d} \Omega^{+}$ $\left(\hat{\partial}_{L}^{i} V_{N}\right)$, and, by FKG and shift invariance, we have

$$
P_{N}^{0}\left(\Omega^{+}\left(\partial_{L} V_{N}\right)\right) \geqq \prod_{i=-d}^{d} P_{N}^{0}\left(\Omega^{+}\left(\hat{\partial}_{L}^{i} V_{N}\right)\right)=\prod_{i=-d}^{d} P_{N}^{i, 0}\left(\Omega^{+}\left(\partial_{L}^{i} V_{N}\right)\right),
$$

and (4.4) will follow from

$$
\liminf _{N \rightarrow \infty} \frac{1}{N^{d-1}} \log P_{N}^{i, 0}\left(\Omega^{+}\left(\partial_{L}^{i} V_{N}\right)\right) \geqq \liminf _{N \rightarrow \infty} \frac{1}{N^{d-1}} \log P^{i, 0}\left(\Omega^{+}\left(\partial_{L}^{i} V_{N}\right)\right),
$$

for each $i=-d, \ldots, d$. For fixed $\delta \in(0,1)$, we have by FKG,

$$
\begin{aligned}
P_{N}^{i, 0}\left(\Omega^{+}\left(\partial_{L}^{i} V_{N}\right)\right) & \geqq P_{N}^{i, 0}\left(\Omega^{+}\left(\partial_{L}^{i} V_{\delta N}\right)\right) P_{N}^{i, 0}\left(X(k) \geqq 0, k \in \partial_{L} V_{N}^{i} \backslash \partial_{L} V_{\delta N}^{i}\right) \\
& \geqq P_{N}^{i, 0}\left(\Omega^{+}\left(\partial_{L}^{i} V_{\delta N}\right)\right) \exp \left(-c_{1}(1-\delta)^{d-1} N^{d-1} L\right)
\end{aligned}
$$

Thus, it suffices to show (4.6) with $\partial_{L} \Omega_{N}^{i,+}$ replaced by $\partial_{L} \Omega_{\delta N}^{i,+}$, for each fixed $\delta \in(0,1)$. Let $F_{N, L}^{i}(X)=\left.\frac{P^{i, 0}(d X)}{P_{N}^{i, 0}(d X)}\right|_{\partial_{L} V_{\delta N}^{i}}$, then, for each $p, q>1$ with $1 / p+1 / q=1$, we have by Hölder's inequality

$$
P^{0, i}\left(\Omega^{+}\left(\partial_{L}^{i} V_{\delta N}\right)\right) \leqq P_{N}^{0, i}\left(\Omega^{+}\left(\partial_{L}^{i} V_{\delta N}\right)\right)^{1 / p}\left\|F_{N, L}^{i}\right\|_{L^{q}\left(P_{N}^{0, l}\right)}
$$


In order to verify (4.6), it is enough to prove that, for each $q>1$,

$$
\limsup _{N \rightarrow \infty} \frac{1}{N^{d-1}} \log \left\|F_{N, L}^{i}\right\|_{L^{q}\left(P_{N}^{0, i}\right)}=0 \text {. }
$$

Let $G_{N, \delta}^{i}$ and $G_{\delta}^{i}$ denote the covariance matrices of $P_{N}^{i, 0}$ and $P^{i, 0}$ restricted to the box $\partial_{L} V_{\delta N}^{i}$, then

$$
\left\|F_{N, L}^{i}\right\|_{L^{q}\left(P_{N}^{0, t}\right)}=\operatorname{det}\left(I+R_{N}\right)^{1 / 2} \operatorname{det}\left(I+q R_{N}\right)^{-1 / 2 q},
$$

where $I$ is the identity on the box $\partial_{L} V_{\delta N}^{i}$ and $R_{N}=G_{N, \delta}^{i}\left(G_{\delta}^{i}\right)^{-1}-I$. Set $\left\|R_{N}\right\|=$ $\sup _{k} \sum_{\ell}\left|R_{N}(k, \ell)\right|$. Then we will show in the Appendix (Lemma A.6) that, for each fixed $L \in \mathbb{R}^{+}$and $\delta \in(0,1)$,

$$
\lim _{N \rightarrow \infty}\left\|G_{N, \delta}^{i}-G_{\delta}^{i}\right\|=0 \quad \text { and } \quad\left\|\left(G_{N, \delta}^{i}\right)^{-1}\right\| \leqq 2, \quad\left\|\left(G_{\delta}^{i}\right)^{-1}\right\| \leqq 2 .
$$

Therefore $\lim _{N \rightarrow \infty}\left\|R_{N}\right\|=0$. Thus, if $\left\{\lambda_{j}(N), j=1, \ldots, k_{\max }\right\} \subset \mathbb{R}$, with $k_{\max }=$ $\left|\partial_{L} V_{\delta N}^{i}\right|$ denote the eigenvalues of the matrix $R_{N}$, then as $\max _{i}\left|\lambda_{i}(N)\right| \leqq\left\|R_{N}\right\|$, we see from the above, for $N$ large enough with $\left\|R_{N}\right\|<1 / q$, that

$$
\begin{aligned}
\frac{1}{N^{d-1}} \log \left\|F_{N, L}^{i}\right\|_{L^{q}\left(P_{N}^{0, i}\right)} & =\frac{1}{2 N^{d-1}} \sum_{j=1}^{k_{\max }} \log \left(1+\lambda_{j}(N)\right)-\frac{1}{2 q N^{d-1}} \sum_{j=1}^{k_{\max }} \log \left(1+q \lambda_{j}(N)\right) \\
& \leqq \frac{k_{\max }}{2 N^{d-1}} \log \left(1+\left\|R_{N}\right\|\right)-\frac{k_{\max }}{2 q N^{d-1}} \log \left(1-q\left\|R_{N}\right\|\right),
\end{aligned}
$$

which yields (4.8).

We now turn to the upper bound (4.5), which will follow from

$$
\limsup _{N \rightarrow \infty} \frac{1}{N^{d-1}} \log P_{N}^{0}\left(\partial_{L} \Omega_{\delta N}^{+}\right) \leqq \sum_{i=-d}^{d} \limsup _{N \rightarrow \infty} \frac{1}{N^{d-1}} \log P_{N}^{0, i}\left(\Omega^{+}\left(\partial_{L}^{i} V_{\delta N}\right)\right),
$$

for each fixed $\delta \in(0,1)$ : Once (4.10) is proved, we can proceed as above using (4.7) and (4.8), interchanging the roles of $P_{N}^{i, 0}$ and $P^{i, 0}$. Note that for $i \neq j$ and large $N \geqq 2 L$,

$$
\operatorname{dist}\left(\hat{\partial}_{L}^{i} V_{\delta N}, \hat{\partial}_{L}^{j} V_{\delta N}\right) \geqq c_{2} \delta N .
$$

Also using the estimate (A.2) in the Appendix one shows the existence of a constant $c_{3}<\infty$ such that for all $R \geqq 2 L$,

$$
\sup _{k \in \partial_{L} V_{N}} \sum_{j \in \partial_{L} V_{N},|j-k| \geqq R} G_{N}(k, j) \leqq c_{3} \frac{L^{3}}{R} .
$$

In view of (4.9), (4.11) and (4.12), we can then apply the hypercontractive estimate derived in the proof of Prop. A.18 of [5] and get

$$
P_{N}^{0}\left(\partial_{L} \Omega_{N}^{+}\right) \leqq P_{N}^{0}\left(\bigcap_{i=-d}^{d} \Omega^{+}\left(\hat{\partial}_{L}^{i} V_{\delta N}\right)\right) \leqq \prod_{i=1}^{d} P_{N}^{i, 0}\left(\Omega^{+}\left(\partial_{L}^{i} V_{\delta N}\right)\right)^{\frac{2}{p\left(c_{2} \delta N\right)}},
$$

where $\lim _{N \rightarrow \infty} p\left(c_{2} \delta N\right)=1$. This implies (4.10) and concludes the proof. 
Lemma 4.13. For each $L \in \mathbb{R}^{+}$and $i=-d, \ldots, d$, we have

$$
\liminf _{N \rightarrow \infty} \frac{1}{N^{d-1}} \log P^{0, i}\left(\Omega^{+}\left(\partial_{L}^{i} V_{N}\right)\right)=\limsup _{N \rightarrow \infty} \frac{1}{N^{d-1}} \log P^{0, i}\left(\Omega^{+}\left(\partial_{L}^{i} V_{N}\right)\right) \equiv-\kappa_{L}^{0}\left(e_{i}\right) .
$$

Proof. We use a simple subadditive argument: Take, for example $i=d$. For $\mathbf{M}=$ $\left(M_{1}, \ldots, M_{d-1}\right) \in \mathbb{N}^{d-1}$ let

$$
\partial_{L}^{d} V_{\mathbf{M}}=\left\{k \in \mathbb{Z}^{d}:-L-1 \leqq k_{d}<0,0 \leqq k_{j} \leqq M_{j}, j=1, \ldots, d-1\right\} .
$$

Then by the FKG property and stationarity of $P^{d, 0}$, we have

$$
P^{d, 0}\left(\Omega^{+}\left(\partial_{L}^{d} V_{\mathbf{M}+\mathbf{M}^{\prime}}\right)\right) \geqq P^{d, 0}\left(\Omega^{+}\left(\partial_{L}^{d} V_{\mathbf{M}}\right)\right) P^{d, 0}\left(\Omega^{+}\left(\partial_{L}^{d} V_{\mathbf{M}^{\prime}}\right)\right),
$$

where $\mathbf{M}, \mathbf{M}^{\prime}$ and $\mathbf{M}+\mathbf{M}^{\prime}$ satisfy $\left(M+M^{\prime}\right)_{j}=M_{j}+M_{j}^{\prime}$ for some $1 \leqq j \leqq d-1$ and $M_{i}=M_{i}^{\prime}=\left(M+M^{\prime}\right)_{i}$ for $i \neq j$. That is, $\mathbf{M} \rightarrow-\log P^{d, 0}\left(\Omega^{+}\left(\partial_{L}^{d} V_{\mathbf{M}}\right)\right)$ is subadditive in each coordinate $M_{j}, j=1, \ldots, d-1$, and therefore the limit

$$
\lim _{N \rightarrow \infty}-\frac{1}{N^{d-1}} \log P^{d, 0}\left(\Omega^{+}\left(\partial_{L}^{d} V_{N}\right)\right)=\inf _{N \in \mathbb{N}}-\frac{1}{N^{d-1}} \log P^{d, 0}\left(\Omega^{+}\left(\partial_{L}^{d} V_{N}\right)\right)=-\kappa_{L}^{0}\left(e_{d}\right)
$$

exists.

Proof of (4.2). By Lemmas 4.3 and 4.13, we know that for each fixed $L \in \mathbb{N}$,

$$
\lim _{N \rightarrow \infty} \frac{1}{N^{d-1}} \log P_{N}^{0}\left(\partial_{L} \Omega_{N}^{+}\right)=\sum_{i=-d}^{d} \lim _{N \rightarrow \infty} \frac{1}{N^{d-1}} \log P^{0, i}\left(\Omega^{+}\left(\partial_{L}^{i} V_{N}\right)\right)=-\sum_{i=-d}^{d} \kappa_{L}^{0}\left(e_{i}\right)
$$

Also $\kappa_{L}^{0}\left(e_{i}\right)$ is increasing in $L$ with $0<\kappa_{1}^{0}\left(e_{i}\right) \leqq \kappa_{L}^{0}\left(e_{i}\right) \leqq K<\infty$, cf. Prop. 2.9. This implies $\lim _{L \rightarrow \infty} \kappa_{L}^{0}\left(e_{i}\right)=\kappa^{0}\left(e_{i}\right) \in(0, \infty)$. Trivially we have

$$
\limsup _{N \rightarrow \infty} \frac{1}{N^{d-1}} \log P_{N}^{0}\left(\Omega_{N}^{+}\right) \leqq \lim _{L \rightarrow \infty} \lim _{N \rightarrow \infty} \frac{1}{N^{d-1}} \log P_{N}^{0}\left(\partial_{L} \Omega_{N}^{+}\right)=-\sum_{i=-d}^{d} \kappa^{0}\left(e_{i}\right) .
$$

On the other hand by FKG,

$$
P_{N}^{0}\left(\Omega_{N}^{+}\right) \geqq P_{N}^{0}\left(\Omega_{(N-L)}^{+}\right) P_{N}^{0}\left(\partial_{L} \Omega_{N}^{+}\right),
$$

where, by Prop. 2.9,

$$
\lim _{L \rightarrow \infty} \liminf _{N \rightarrow \infty} \frac{1}{N^{d-1}} \log P_{N}^{0}\left(\Omega_{(N-L)}^{+}\right)=0 .
$$

This shows

$$
\liminf _{N \rightarrow \infty} \frac{1}{N^{d-1}} \log P_{N}^{0}\left(\Omega_{N}^{+}\right) \geqq-\lim _{L \rightarrow \infty} \sum_{i=-d}^{d} \kappa_{L}^{0}\left(e_{i}\right)=-\sum_{i=-d}^{d} \kappa^{0}\left(e_{i}\right),
$$

and concludes the proof.

Remark 4.14. Note that we can view $\hat{P}^{i, 0}$ as a Gaussian field on $\Omega_{i} \equiv\left(\mathbb{R}^{\mathbb{Z}^{-}}\right)^{\mathbb{Z}^{d-1}}$, invariant under the shift on $\mathbb{Z}^{d-1}$. Set $P_{N, L}^{i,+} \equiv P_{N}^{i, 0}\left(\cdot \mid \Omega^{+}\left(\partial_{L}^{i} V_{N}\right)\right)$, Then, in view of 
(3.2) and (3.9) we have

$$
\lim _{L \rightarrow \infty} \limsup _{N \rightarrow \infty} E_{N}^{i, 0}\left[X(k) \mid \partial_{L} \Omega_{N}^{i,+}\right] \leqq \sqrt{c \log \left|k_{i}\right|}, \quad k \in \mathbb{Z}_{i}^{d}
$$

for some constant $c<\infty$ and thus $\left\{P_{N, L}^{i,+}: N \geqq L \in \mathbb{N}\right\}$ is tight. Using the monotonicity (3.3), or a Gibbsian characterization, we can then show the weak convergence on $\Omega_{i}$

$$
\lim _{L \rightarrow \infty} \lim _{N \rightarrow \infty} P_{N, L}^{i,+}=P^{i,+},
$$

for some $P^{i,+}$, stationary with respect to the shift on $\mathbb{Z}^{d-1}$.

Remark 4.16. Let $\omega \in S^{d-1}$ be a unit vector and set $\mathbb{Z}_{\omega}^{d}=\left\{k \in \mathbb{Z}^{d}: \omega \cdot k<0\right\}$,

$$
\partial_{L}^{\omega} V_{N}=\left\{k \in \mathbb{Z}^{d}:-L-1 \leqq k \cdot \omega<0,\left|k \cdot \tilde{e}_{j}\right| \leqq N, j=1, \ldots, d-1\right\},
$$

where $\left(\omega, \tilde{e}_{1}, \ldots, \tilde{e}_{d-1}\right)$ is an orthonormal basis of $\mathbb{R}^{d}$. Next, for fixed $\alpha \in \mathbb{R}$, let $P^{\omega, \alpha}=P^{\alpha}\left(\cdot \mid X(j)=\alpha, j \notin \mathbb{Z}_{\omega}^{d}\right)$, then using the same arguments as above, one shows that the limits

$$
\lim _{N \rightarrow \infty} \frac{1}{N^{d-1}} \log P^{\omega, \alpha}\left(\Omega^{+}\left(\partial_{L}^{\omega} V_{N}\right)\right)=-\kappa_{L}^{\alpha}(\omega), \quad \kappa^{\alpha}(\omega) \equiv \lim _{L \rightarrow \infty} \kappa_{L}^{\alpha}(\omega)
$$

exist and do not depend on the choice of the basis $\left\{\tilde{e}_{1}, \ldots, \tilde{e}_{d-1}\right\}$. Moreover

$$
0<\inf _{\omega \in S^{d-1}} \kappa^{\alpha}(\omega)<\sup _{\omega \in S^{d-1}} \kappa^{\alpha}(\omega)<\infty
$$

Next consider a bounded open domain $\Lambda$ in $\mathbb{R}^{d}$ with polygonal boundary $\partial \Lambda=\bigcup_{i=1}^{\Lambda} \partial_{i} \Lambda$ and set $\Lambda_{N}=N \Lambda \cap \mathbb{Z}^{d}$. Then a simple modification of the proof of Theorem 4.1 yields

$$
\begin{aligned}
\lim _{N \rightarrow \infty} \frac{1}{N^{d-1}} \log P_{\Lambda_{N}}^{\alpha}\left(\Omega^{+}\left(\Lambda_{N}\right)\right) & =\lim _{L \rightarrow \infty} \lim _{N \rightarrow \infty} \frac{1}{N^{d-1}} \log P_{\Lambda_{N}}^{\alpha}\left(\Omega^{+}\left(\partial_{L} \Lambda_{N}\right)\right) \\
& =-\sum_{i=1}^{\Delta} \kappa^{\alpha}\left(n_{i}\right)\left|\partial_{i} \Lambda\right|
\end{aligned}
$$

where $n_{i}$ is the unit normal of $\partial_{i} \Lambda$ and $\left|\partial_{i} \Lambda\right|$ is the area.

Note that in the derivation of the above limit, one uses explicitly the fact that the pieces of boundary $\partial_{i} \Lambda$ are flat in the above argument. In particular a generalization as suggested by $D$. Ioffe:

$$
\lim _{N \rightarrow \infty} \frac{1}{N^{d-1}} \log P_{N}^{\alpha}\left(\Omega^{+}\left(\Lambda_{N}\right)\right)=-\int_{\partial \Lambda} \kappa^{\alpha}\left(n_{x}\right) d x,
$$

where $\Lambda$ is an open domain of $\mathbb{R}^{d}$ with piecewise smooth boundary is not immediate. 


\section{Appendix}

The object of this Appendix is to derive some useful estimates for the covariance matrices, based on the random walk representation. The basic estimate is the following

Lemma A.1. There exists a constant $c_{1}<\infty$, such that for all $k, j \in \partial_{L} V_{N}$,

$$
G_{N}(k, j) \leqq \begin{cases}c_{1}|k-j|^{2-d} & 1 \leqq|k-j|<2 L, \\ c_{1} L^{2}|k-j|^{-d} & |k-j| \geqq 2 L .\end{cases}
$$

In particular,

$$
\sup _{k \in \partial_{L} V_{N}} \sum_{j \in \partial_{L} V_{N}} G_{N}(k, j) \leqq c_{2} L^{2} .
$$

Proof. First note that $G_{N}(k, j) \leqq G(k, j)$, where

$$
\lim _{|k-j| \rightarrow \infty} G(k, j)|k-j|^{d-2}=\frac{\Gamma(d / 2) d^{(3-d) / 2}}{(d-2) \pi^{d / 2}},
$$

cf. Sect. 26 of [12]. This shows (A.2) for $|k-j|<2 L$. Next take $k, j$ with $|k-j| \geqq$ $2 L$. As above, let us move the boundary of $V_{N}$ to the origin. Thus suppose that $k, j \in \partial_{L}^{d} V_{N}$ with $-L-1 \leqq j_{d} \leqq k_{d}<0$. Then, by the reflection principle,

$$
G_{N}^{d}(k, j) \leqq G^{d}(k, j) \leqq G^{d}\left(k^{\prime}, j\right)=G\left(k^{\prime}, j\right)-G\left(k^{\prime}, \hat{j}\right)
$$

where $k^{\prime}=\left(k_{1}, \ldots, k_{d-1}, j_{d}\right), \hat{j}=\left(j_{1}, \ldots, j_{d-1},-j_{d}\right)$. We claim that

$$
\limsup _{\left|k^{\prime}-j\right| \rightarrow \infty}\left|k^{\prime}-j\right|^{d} G^{d}\left(k^{\prime}, j\right) \leqq c_{3}\left|j_{d}\right|^{2}
$$

This will imply the second inequality in (A.2). In order to prove (A.5), we use harmonic analysis as in Sect. 7 of [12]: let $\hat{Q}_{\mathrm{d}}(\theta)=\sum_{k \in \mathbb{Z}^{d}} Q_{\mathrm{d}}(0, k) e^{i k} \cdot 0=$ $\frac{1}{d} \sum_{j=1}^{d} \cos \left(\theta_{j}\right)$, then

$$
\begin{aligned}
G^{d}\left(k^{\prime}, j\right) & =\frac{1}{(2 \pi)^{d}} \int_{(-\pi, \pi]^{d}}\left(e^{-i\left(k^{\prime}-j\right) \cdot \theta}-e^{-i\left(k^{\prime}-\hat{j}\right) \cdot \theta}\right) \psi(\theta) d \theta \\
& =\frac{1}{(2 \pi)^{d}} \int_{(-\pi, \pi]^{d}} e^{-i\left(k^{\prime}-j\right) \cdot \theta}\left(1-\cos \left(2 j_{d} \theta_{d}\right)\right) \psi(\theta) d \theta \\
& =\frac{\Delta^{-d}}{(2 \pi)^{d}} \int_{(-\pi \Delta, \pi \Delta]^{d}} e^{-i \frac{\left(k^{\prime}-j\right)}{\Delta} \cdot \theta}\left(1-\cos \left(2 \frac{j_{d}}{\Delta} \theta_{d}\right)\right) \psi\left(\frac{\theta}{\Delta}\right) d \theta,
\end{aligned}
$$

where $\psi(\theta)=\frac{1}{1-\hat{Q}_{\mathrm{d}}(\theta)}$ and $\Delta=\left|k^{\prime}-j\right|$. Thus, by (A.4) and rotation invariance

$$
\limsup _{\Delta \rightarrow \infty} \Delta^{d} G_{N}^{d}\left(k^{\prime}, j\right) \leqq \frac{4 d\left|j_{d}\right|^{2}}{(2 \pi)^{d}} \int_{\mathbb{R}^{d}} e^{-i \theta_{1}} \frac{\left|\theta_{d}\right|^{2}}{|\theta|^{2}} d \theta<\infty .
$$

Finally, (A.3) follows from (A.2). 
Next, recall the definitions of $G_{N, \delta}^{i}$ and $G_{\delta}^{i}$ : the covariance matrices of $P_{N}^{i, 0}$ and $P^{i, 0}$ restricted to the box $\partial_{L}^{i} V_{\delta N}$, cf. Proof of Lemma 4.3.

Lemma A.6. For each fixed $L>0$ and $\delta \in(0,1)$ we have

$$
\lim _{N \rightarrow \infty}\left\|G_{N, \delta}^{i}-G_{\delta}^{i}\right\|=0 \text {. }
$$

Moreover

$$
\left\|\left(G_{N, \delta}^{i}\right)^{-1}\right\| \leqq 2, \quad\left\|\left(G_{\delta}^{i}\right)^{-1}\right\| \leqq 2 .
$$

Proof. Using the random walk representation, we have

$$
\sum_{j \in \partial_{L}^{i} V_{\delta N}}\left|G^{i}(k, j)-G_{N}^{i}(k, j)\right|=\mathbb{E}_{k}\left[\sum_{n=\tau_{N}^{i}+1}^{\tau^{i}} 1_{\partial_{L}^{i} V_{\delta N}}\left(\xi_{n}\right) ; \tau_{N}^{i}<\tau^{i}\right] .
$$

Thus, by the strong Markov property of the random walk and (A.3),

$$
\begin{aligned}
\sum_{j \in \partial_{L}^{i} V_{\delta N}}\left|G_{N}^{i}(k, j)-G^{i}(k, j)\right| & =\mathbb{E}_{k}\left[\mathbb{E}_{\xi_{\tau_{N}^{i}}}\left[\sum_{n=+1}^{\tau^{i}} 1_{\partial_{L} V_{\delta N}^{i}}\left(\xi_{n}\right)\right] ; \xi_{\tau_{N}^{i}} \notin \mathbb{Z}_{i}^{d}\right] \\
& \leqq c_{1} L^{2} \mathbb{P}_{k}\left(\xi_{\tau_{N}^{l}} \notin \mathbb{Z}_{i}^{d}\right)=c_{1} L^{2} \mathbb{P}_{k}\left(\tau_{N}^{i}<\tau^{i}\right)
\end{aligned}
$$

This implies (A.7), since

$$
\lim _{N \rightarrow \infty} \sup _{k \in \partial_{L}^{i} V_{\delta N}} \mathbb{P}_{k}\left(\tau_{N}<\tau_{N}^{i}\right)=0
$$

Next note that $\left\langle f,\left(G_{N, \delta}^{i}\right)^{-1} f\right\rangle_{\partial_{L}^{l} V_{\delta N}}$ and $\left\langle f,\left(G_{\delta}^{i}\right)^{-1} f\right\rangle_{\partial_{L}^{l} V_{\delta N}}, f \in \ell^{2}\left(\partial_{L}^{i} V_{\delta N}\right)$, are the Dirichlet forms associated with the simple random walk embedded in the box $\partial_{L}^{i} V_{\delta N}$ and killed as it exits $V_{N}^{i}$ and $\mathbb{Z}_{i}^{d}$ respectively. That is, if $\tilde{\tau}=\inf \{n \geqq 1$ : $\left.\xi_{n} \in \partial_{L}^{i} V_{\delta N}\right\}$, then $\left(G_{N, \delta}^{i}\right)^{-1}(k, k)=\left(G_{\delta}^{i}\right)^{-1}(k, k)=1$ and, for $j \neq k$,

$$
\left(G_{N, \delta}^{i}\right)^{-1}(k, j)=-\mathbb{P}_{k}\left(\xi_{\tilde{\tau}}=j ; \tilde{\tau}<\tau_{N}^{i}\right),\left(G_{\delta}^{i}\right)^{-1}(k, j)=-\mathbb{P}_{k}\left(\xi_{\tilde{\tau}}=j ; \tilde{\tau}<\tau^{i}\right) .
$$

Therefore

$$
\begin{gathered}
\sum_{j \in \partial_{L}^{l} V_{\delta N}}\left|\left(G_{N, \delta}^{i}\right)^{-1}(k, j)\right| \leqq 1+\mathbb{P}_{k}\left(\tilde{\tau}<\tau_{N}^{i}\right) \leqq 2 \\
\sum_{j \in \partial_{L}^{i} V_{\delta N}}\left|\left(G_{\delta}^{i}\right)^{-1}(k, j)\right| \leqq 1+\mathbb{P}_{k}\left(\tilde{\tau}<\tau^{i}\right) \leqq 2
\end{gathered}
$$

which shows (A.8).

Finally, let us prove that a random walk starting at distance $L_{N}$ from the boundary of the box $V_{N}$, is more likely to get trapped in a sub-lattice of mesh $L_{N}^{2 / d}$, before it exits $V_{N}$. More precisely

Lemma A.9. Let $\Delta_{N}=\left[L_{N}^{2 / d}\right]$, and set

$$
\Lambda_{N}(\varepsilon) \equiv\left[\varepsilon \Delta_{N}\right] \mathbb{Z}^{d} \cap\left\{k: \frac{L_{N}}{2} \leqq \operatorname{dist}\left(k, V_{N}^{\complement}\right) \leqq \frac{5 L_{N}}{2}\right\} .
$$

Let $\tau_{\varepsilon}=\inf \left\{n \in \mathbb{N}_{0}: \xi_{n} \in \Lambda_{N}(\varepsilon)\right\}$, then we can choose $\varepsilon>0$ independently of $N$, such that

$$
\inf _{k \in W_{N}\left(L_{N}\right)} \mathbb{P}_{k}\left(\tau_{\varepsilon}<\tau_{N}\right) \geqq \frac{1}{2}
$$


Proof. Let $\tilde{\tau}_{N}=\inf \left\{n \geqq 0: \operatorname{dist}\left(\xi_{n}, V_{N}^{\complement}\right) \notin\left[\frac{L_{N}}{2}, \frac{5 L_{N}}{2}\right]\right\}$, then

$$
\mathbb{P}_{k}\left(\tau_{N} \leqq \tau_{\varepsilon}\right) \leqq \mathbb{P}_{k}\left(\tilde{\tau}_{N} \leqq \tau\left(\left[\varepsilon \Delta_{N}\right]\right)\right) \leqq \mathbb{P}_{k}\left(\tilde{\tau}_{N} \leqq \varepsilon L_{N}^{2}\right)+\mathbb{P}_{k}\left(\tau\left(\left[\varepsilon \Delta_{N}\right]\right) \geqq \varepsilon L_{N}^{2}\right)
$$

Note that $\sup _{k \in W_{N}\left(L_{N}\right)} \mathbb{P}_{k}\left(\tilde{\tau}_{N} \leqq \varepsilon L_{N}^{2}\right)$ converges to 0 as $\varepsilon \searrow 0$, uniformly in $N$. On the other hand by (A.8) of [3],

$$
\sup _{k \in W_{N}\left(L_{N}\right)} \mathbb{P}_{k}\left(\tau\left(\left[\varepsilon \Delta_{N}\right]\right) \geqq \varepsilon L_{N}^{2}\right) \leqq \exp \left(-c_{1} \frac{\varepsilon L_{N}^{2}}{\left(\varepsilon L_{N}^{2 / d}\right)^{d}}\right)=\exp \left(-c_{1} \varepsilon^{1-d}\right)
$$

Acknowledgements. Special thanks are due to Erwin Bolthausen for valuable suggestions. In particular, the idea of the conditioning argument in Prop. 2.9 is due to him.

\section{References}

1. Ben Arous, G., Deuschel, J.-D.: The construction of the $d+1$-dimensional Gaussian droplet. Commun. Math. Phys. 179, 467-488 (1996)

2. Bricmont, J., El-Mellouki, A., Fröhlich, J.: Random surfaces in statistical mechanics: Roughening, rounding, wetting. J. Stat. Phys. 42, 743-396 (1986)

3. Bolthausen, E., Deuschel, J.-D.: Critical large deviations for Gaussian fields in the phase transition regime. I. Ann. of Probab. 21, 1876-1920 (1993)

4. Bolthausen, E., Deuschel, J.-D.: Entropic repulsion for the lattice free field, III. The 2-dimensional case. Preprint (1996)

5. Bolthausen, E., Deuschel, J.-D., Zeitouni, O.: Entropic repulsion for the lattice free field. Commun. Math. Phys. 170, 417-443 (1995)

6. de Coninck, J., Dunlop, F., Rivasseau, V.: On the microscopic validity of the Wulff construction and of the Young equation. Commun. Math. Phys. 121, 401-415 (1989)

7. van Enter, A.C.D., Fernandez, R., Sokal, A.D.: Regularity properties and pathologies of position-space renormalization-group transformations: Scope and limitations of Gibbsian theory. J. Stat. Phys. 72, 879-1169 (1993)

8. Georgii, H.-O.: Gibbs measures and phase transitions. Berlin: de Gruyter, 1988

9. Herbst, I., Pitt, L.: Diffusion equation techniques in stochastic monotonicity and positive correlations. Prob. Th. Rel. Fields 87, 275-312 (1991)

10. Bolthausen, E., Ioffe, D.: Harmonic cristal on the wall: A microscopic approach. Preprint (1996)

11. Lebowitz, J.L. and Maes, C.: The effect of an external field on an interface, entropy repulsion. J. Stat. Phys. 46, 39-49 (1987)

12. Spitzer, F.: Principles of random walk. 2nd ed. New York: Springer, 1976 
